\title{
Detection of Incomplete Bladder Duplication by SPECT/CT
}

\author{
Yingzhuo Yang*, Wen Yang, Qiao Wang, and Yun Duan* \\ Department of Nuclear Medicine, Sichuan Cancer Hospital and Institute, School of Medicine, University of Electronic Science and \\ Technology of China, Chengdu, China
}

Bladder duplication is an extremely rare congenital urinary tract malformation that is often discovered incidentally. Here, we report a case of incomplete bladder duplication diagnosed by SPECT/CT in a 65-y-old man with lung cancer. Compared with the results of whole-body planar bone scintigraphy, this SPECT/CT finding caused the diagnosis to be revised in this patient with suspected bone metastases. To our knowledge, this is the first documented case of incomplete bladder duplication discovered by SPECT/CT.

Key Words: SPECT/CT; bladder duplication; bone metastasis J Nucl Med Technol 2020; 48:381-383 DOI: 10.2967/jnmt.120.246801

\section{B}

ladder duplication is a rare congenital urinary tract malformation, and to date, only about 100 cases have been reported worldwide (1). Patients with bladder duplication may complain of incontinence, recurrent infections, and bladder outlet obstruction; however, most patients are asymptomatic. Some cases are discovered incidentally during voiding cystourethrography, whereas others are discovered on autopsy (2). We incidentally discovered a case of incomplete bladder duplication on SPECT/CT in a patient with lung cancer. This might be the first report of incomplete bladder duplication discovered by SPECT/CT and could provide a chance for urologists to learn more about bladder duplication and improve their diagnostic ability.

\section{CASE REPORT}

This case study was approved by the Institutional Ethics Committee of the Sichuan Cancer Hospital and Institute and is in accordance with the 1964 Declaration of Helsinki and its later amendments or comparable ethical standards.

Received Apr. 9, 2020; revision accepted Jun. 18, 2020.

For correspondence or reprints contact: Yun Duan, Sichuan Cancer Hospital and Institute, No. 55, 4th Section of South Ren-Min Rd., Chengdu 610041, China.

E-mail: duanyuncd@163.com

${ }^{*}$ Contributed equally to this work.

Published online Jul. 24, 2020.

COPYRIGHT (c) 2020 by the Society of Nuclear Medicine and Molecular Imaging.
The patient provided written informed consent to the publication of this case report.

A 65-y-old man with a 1-mo history of cough, hemoptysis, and chest pain was diagnosed with squamous cell carcinoma of the left lung. He had no pain elsewhere in his body. He had a history of hypertension and hepatitis but not of trauma. Pretreatment whole-body planar bone scintigraphy was performed to detect bone metastases. There were 2 suggestive foci of uptake on the planar bone scintigraphy image - one at the lateral aspect of the right seventh rib and the other one at the pelvic level. The focal uptake at the

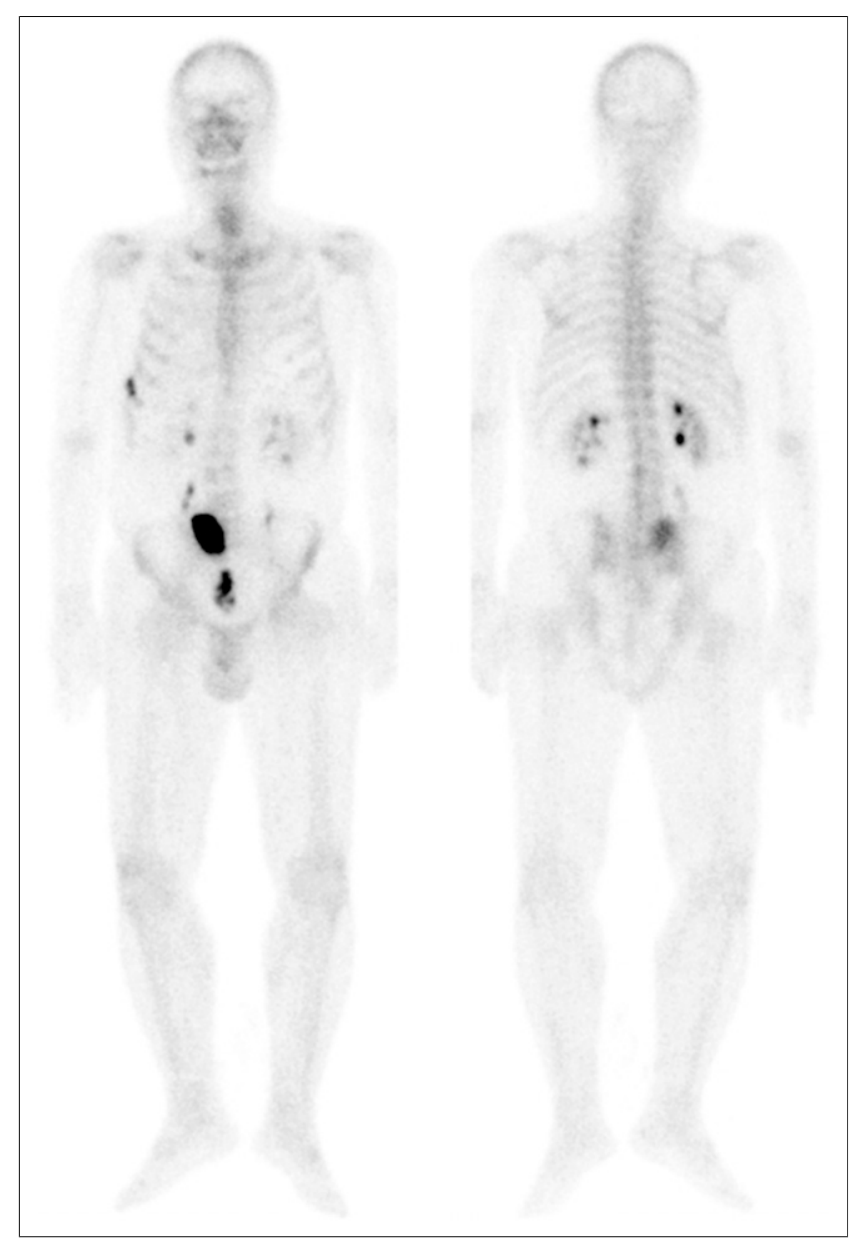

FIGURE 1. Whole-body planar scintigraphy image with 2 suggestive foci of uptake. 


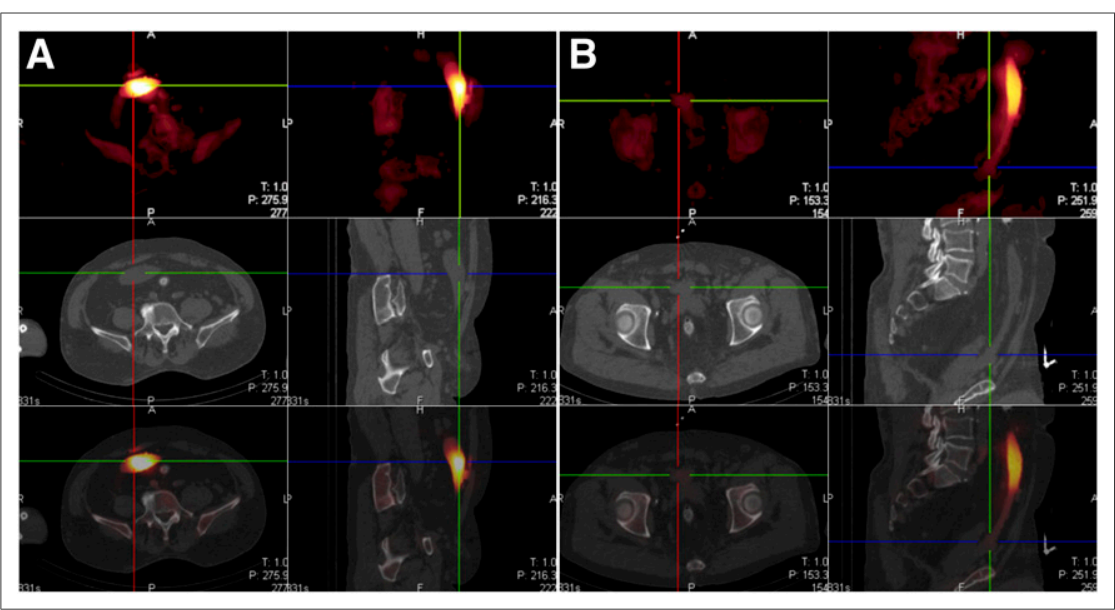

FIGURE 2. SPECT/CT images showing high uptake in abdominal cavity corresponding to soft tissue.

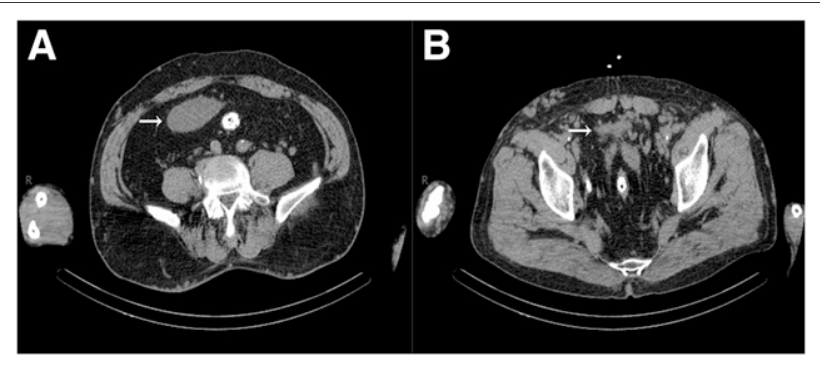

FIGURE 3. CT scan showing focal abdominal uptake suggestive of bladder tissue.

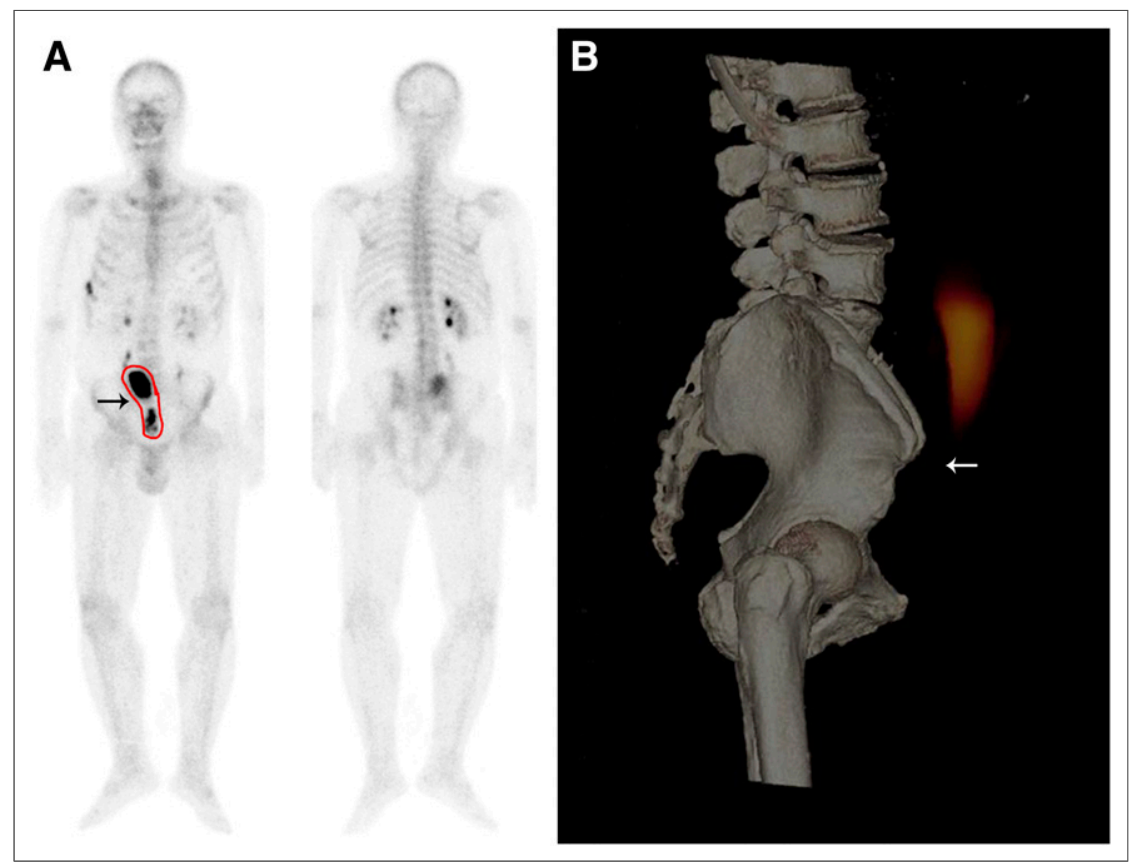

FIGURE 4. (A) Planar bone scintigraphy image showed gourd-shaped radiotracer uptake (arrow). (B) Fused SPECT/CT image showing the bladder was not clearly visible in the pelvis.

\section{DISCUSSION}

pelvic level was more obvious on the anterior image than on the posterior image (Fig. 1). SPECT/CT was performed to localize and characterize the pelvic uptake. Axial CT and SPECT/ CT showed that it corresponded to soft tissue, not bone (Fig. 2). CT was then performed, and the appearance of the uptake in the soft-tissue window was suggestive of bladder tissue (Fig. 3). The planar bone scintigraphy image showed the uptake to be gourd-shaped, consistent with a duplicated bladder. Thus, we asked the patient to void before the SPECT/CT acquisition. The resulting 3-dimensional fusion image showed the bladder was not clearly visible in the pelvis (Fig. 4).

This report describes a case of incomplete bladder duplication that was detected incidentally on SPECT/CT performed to assess and characterize abnormal uptake in a patient with squamous cell carcinoma of the left lung.

Bladder duplication is rarely solitary and usually includes a duplicated urethra and, in $90 \%$ of cases, duplicated external genitalia (3). This disease can be divided into many clinical subtypes, including complete and incomplete, sagittal plane and coronal plane, cystic bladder, and gourd-shaped bladder, among others $(2,4)$. Bladder duplication is a rare anomaly. Complete bladder duplication is the most common type, with more than 50 reported cases, whereas there are only 10 reported cases of incomplete bladder duplication (5). Bladder duplication is more common in the sagittal plane than in the coronal plane, with a ratio of 2.5:1 among the reported cases (5).

Bladder duplication can vary from duplication of the intravesicular septum to complete duplication of the lower urinary tract $(3-5)$. The clinical significance of lower urinary tract duplication is not clear. Bladder duplication does not always require surgical management (5). Intervention might be necessary to eliminate stasis and achieve continence. Ultrasonography or an intravenous urogram usually reveals associated upper-tract abnormalities. However, treatment is not necessary if bladder function and drainage are satisfactory (6).

In this case, the addition of SPECT/ $\mathrm{CT}$ improved diagnostic confidence over that with SPECT or planar bone scintigraphy alone. To our knowledge, this is 
the first reported case of bladder duplication discovered by SPECT/CT. SPECT/CT helped us to understand and characterize the focal uptake and, thus, to revise the original diagnosis. Exclusion of bone metastasis had a significant impact on this patient.

Unfortunately, the patient discontinued treatment. Therefore, further imaging data could not be obtained to clarify whether this patient had urethra duplication as well.

\section{CONCLUSION}

Bladder duplication is a rare urologic complication. This unique case provides a chance for urologists to learn more about bladder duplication and improve their diagnostic ability.

\section{DISCLOSURE}

No potential conflict of interest relevant to this article was reported.

\section{ACKNOWLEDGMENTS}

We thank Editage (www.editage.cn) for English language editing.

\section{REFERENCES}

1. Goh DW, Davey RB, Dewan PA. Bladder, urethral, and vaginal duplication. $J$ Pediatr Surg. 1995;30:125-126.

2. O'Sullivan GJ, Hunter J, Dubbins PA. Case report: antenatal ultrasonic diagnosis of complete bladder duplication. Clin Radiol. 1996;51:818819.

3. Okur H, Keskin E, Zorludemir U. Tubular duplication of the hindgut with genitourinary anomalies. J Pediatr Surg. 1992;27:1239-1240.

4. Dunetz GN, Bauer SB. Complete duplication of bladder and urethra. Urology. 1985;25:179-182.

5. Evangelidis A, Murphy JP, Gatti JM. Incomplete bladder duplication presenting antenatally. Urology. 2004;64:589-592.

6. Mirshemirani AR, Sadeghyian N, Mohajerzadeh L, et al. Diphallus: report on six cases and review of the literature. Iran J Pediatr. 2010;20:353357. 